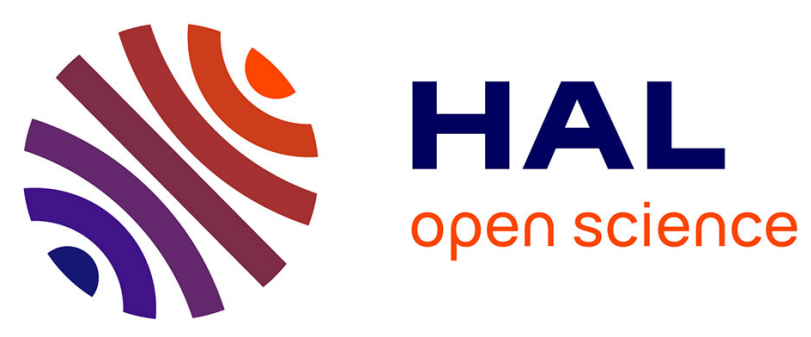

\title{
Bevacizumab Maintenance Versus No Maintenance During Chemotherapy-Free Intervals in Metastatic Colorectal Cancer: A Randomized Phase III Trial (PRODIGE 9)
}

Thomas Aparicio, Francois Ghiringhelli, Valérie Boige, Karine Le Malicot, Julien Taieb, Olivier Bouché, Jean-Marc Phelip, Eric François, Christian Borel, Roger Faroux, et al.

\section{To cite this version:}

Thomas Aparicio, Francois Ghiringhelli, Valérie Boige, Karine Le Malicot, Julien Taieb, et al.. Bevacizumab Maintenance Versus No Maintenance During Chemotherapy-Free Intervals in Metastatic Colorectal Cancer: A Randomized Phase III Trial (PRODIGE 9). Journal of Clinical Oncology, 2018, 36 (7), pp.674 - 681. 10.1200/JCO.2017.75.2931 . hal-01728824

\section{HAL Id: hal-01728824 \\ https://u-bourgogne.hal.science/hal-01728824}

Submitted on 1 Jun 2021

HAL is a multi-disciplinary open access archive for the deposit and dissemination of scientific research documents, whether they are published or not. The documents may come from teaching and research institutions in France or abroad, or from public or private research centers.
L'archive ouverte pluridisciplinaire HAL, est destinée au dépôt et à la diffusion de documents scientifiques de niveau recherche, publiés ou non, émanant des établissements d'enseignement et de recherche français ou étrangers, des laboratoires publics ou privés. 
Author affiliations and support information (if applicable) appear at the end of this article.

Published at jco.org on January 18, 2018. C.L. and J.B. participated equally. Clinical trial information: NCT00952029 Corresponding author: Thomas Aparicio, MD, PhD, Gastroenterology and Digestive Oncology, Saint Louis Hospital, AP-HP, Université Paris 7, Sorbonne Paris Cité, 1 Ave Claude Vellefaux, 75010 Paris, France; e-mail: thomas.aparicio@aphp.fr. (C) 2018 by American Society of Clinical Oncology

$0732-183 X / 18 / 3607 w-674 w / \$ 20.00$

\section{Bevacizumab Maintenance Versus No Maintenance During Chemotherapy-Free Intervals in Metastatic Colorectal Cancer: A Randomized Phase III Trial (PRODIGE 9)}

Thomas Aparicio, Francois Ghiringhelli, Valérie Boige, Karine Le Malicot, Julien Taieb, Olivier Bouché, Jean-Marc Phelip, Eric François, Christian Borel, Roger Faroux, Laetitia Dahan, Stéphane Jacquot, Dominique Genet, Faiza Khemissa, Etienne Suc, Françoise Desseigne, Patrick Texereau, Come Lepage, Jaafar Bennouna, and PRODIGE 9 Investigators

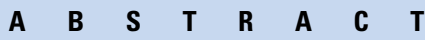

\section{Purpose}

Conflicting results are reported for maintenance treatment with bevacizumab during chemotherapyfree intervals (CFI) in metastatic colorectal cancer after induction chemotherapy.

\section{Patients and Methods}

In this open-label, phase III, randomized controlled trial, we compared the tumor control duration (TCD) observed with bevacizumab maintenance and with no treatment (observation) during CFI subsequent to induction chemotherapy with 12 cycles of fluorouracil, leucovorin, and irinotecan plus bevacizumab. After disease progression, the induction regimen was repeated for eight cycles, followed by a new CFI.

\section{Results}

From March 2010 to July 2013, 491 patients were randomly assigned. Disease progression or death occurred during induction chemotherapy in 85 patients (17\%); 261 patients (53\%) had at least one reinduction, $107(22 \%)$ had two reinductions, and $56(11 \%)$ had three or more reinductions. The median TCD was 15 months in both groups; the median progression-free survival (PFS) from randomization was 9.2 and 8.9 months in the maintenance group and observation groups, respectively. The TCD observed in both groups was higher compared with the TCD hypotheses of the trial. The median overall survival (OS) was 21.7 and 22.0 months in the maintenance and observation groups, respectively. In the per-protocol population, defined as patients with at least one reinduction after the first progression, the median duration of the first CFI was 4.3 months in both arms; the median TCD was 17.8 and 23.3 months ( $P=.339$ ), the median PFS was 9.9 and 9.5 months, and the median OS was 27.6 and 28.5 months in the maintenance and observation groups, respectively. Multivariable analysis revealed that female gender, WHO performance status $\geq 2$, and unresected primary tumors were associated with a shorter TCD.

\section{Conclusion}

Bevacizumab maintenance monotherapy did not improve TCD, CFI duration, PFS, or OS.

\section{J Clin Oncol 36:674-681. (C) 2018 by American Society of Clinical Oncology}

\section{INTRODUCTION}

The prognosis of patients with metastatic colorectal cancer (mCRC) has been significantly improved by the use of several chemotherapy drugs, regardless of the sequence. ${ }^{1}$ The addition of bevacizumab to first-line irinotecan plus fluorouracil chemotherapy resulted in a prolonged overall survival (OS) ${ }^{2}$ and became a standard of care in mCRC. The duration of first-line combination chemotherapy is a matter of controversy because of the toxicity that occurs with prolonged treatment. The introduction of chemotherapyfree intervals (CFIs) was proposed in different studies with oxaliplatin- or irinotecan-based first-line chemotherapy. ${ }^{3-5}$ More recently, several studies evaluated maintenance treatment with bevacizumab alone or in combination with fluoropyrimidine after induction chemotherapy. ${ }^{6-9}$ All these studies, except one, investigated maintenance treatment or CFI without any treatment after oxaliplatin-based induction chemotherapy. Only the study by Koeberle et $\mathrm{al}^{8}$ included some 
patients treated with irinotecan-based induction chemotherapy. The primary end points varied across the studies, and none of them showed any difference in OS with respect to different strategies, except in the results of subgroup analysis. Maintenance with bevacizumab alone is attractive because it is a well-tolerated treatment in monotherapy. Nevertheless, previous results for bevacizumab used alone as maintenance therapy are conflicting. One study demonstrated the noninferiority of bevacizumab alone compared with fluoropyrimidine combined with bevacizumab with respect to the time to failure of the strategy, but did not show any improvement in the time to failure of the strategy when bevacizumab was compared with no treatment. ${ }^{7}$ Another study failed to demonstrate the noninferiority of bevacizumab alone compared with continuation of induction chemotherapy with respect to progression-free survival (PFS) ${ }^{6}$ However, bevacizumab monotherapy failed to show efficacy when given after front-line chemotherapy with irinotecan and a fluoropyrimidine. ${ }^{10}$ In the randomized phase III PRODIGE 9 study, we aimed to assess the tumor control duration (TCD) with bevacizumab maintenance treatment or no treatment after irinotecan-based induction chemotherapy combined with bevacizumab.

\section{PATIENTS AND METHODS}

\section{Patient Selection and Study Design}

This open-label, randomized, multicenter, phase III study was conducted by the Fédération Francophone de Cancérologie Digestive and the PRODIGE (Partenariat de Recherche en Oncologie DIGEstive) intergroup in 66 French centers. The study design has been previously published, ${ }^{11}$ and the protocol is provided in the Data Supplement. Main eligible criteria were histologically proven, nonresectable mCRC, WHO status $\leq 2$, life-expectancy $\geq 3$ months, and absence of previous chemotherapy or antiangiogenic therapy for metastatic disease. The study was approved by the Committee for the Protection of Persons Ile de France VIII. The trial was registered on ClinicalTrials.gov (NCT00952029).

Patients were randomly assigned to either induction chemotherapy plus bevacizumab followed by bevacizumab monotherapy in each CFI (maintenance arm) or to induction chemotherapy plus bevacizumab followed by no treatment in each CFI (observation arm). Patients were randomly assigned between the two treatment groups according to a ratio of one to one and using minimization techniques, taking into account the following stratification factors: site, previous primary tumor resection, and Köhne scoring (low versus intermediate versus high). ${ }^{12}$

\section{Treatment Plan and Evaluation}

Induction chemotherapy consisted of 12 cycles of fluorouracil, leucovorin, and irinotecan (FOLFIRI; irinotecan $180 \mathrm{mg} / \mathrm{m}^{2}$, leucovorin $400 \mathrm{mg} / \mathrm{m}^{2}$, fluorouracil bolus $400 \mathrm{mg} / \mathrm{m}^{2}$ followed by fluorouracil $2,400 \mathrm{mg} / \mathrm{m}^{2}$ in continuous infusion for 46 hours) plus bevacizumab $5 \mathrm{mg} / \mathrm{kg}$ every 2 weeks. The disease status was assessed by computed tomography scan every 8 weeks and by clinical evaluation every 2 weeks. At the end of the induction treatment, patients with stable disease or with a tumor response according to Response Evaluation Criteria in Solid Tumors (RECIST) criteria were either treated with bevacizumab monotherapy $5 \mathrm{mg} / \mathrm{kg}$ every 2 weeks (maintenance arm) or received no antitumor treatment (observation arm). When progression according to RECIST criteria occurred during the CFI, a sequence of eight cycles of FOLFIRI plus bevacizumab was reintroduced. If disease control had been achieved with these eight cycles, they were followed by another CFI (with bevacizumab monotherapy in the maintenance arm or no treatment in the observation arm). These sequences were repeated until progression occurred during chemotherapy. Throughout the entire treatment, including the chemotherapy sequence and the CFI, clinical and radiologic assessment was performed every 8 weeks or at any time progression was suspected. Safety was evaluated by means of laboratory and clinical testing before each cycle, during chemotherapy sequences, and before each bevacizumab infusion (maintenance arm) or every 8 weeks (observation arm) during CFIs. Adverse events were graded according to the National Cancer Institute Common Toxicity Criteria, version 2.0. KRAS, NRAS, and BRAF status was recorded after inclusion on the basis of determinations performed locally. Quality of life (QOL) was assessed using the Quality of Life Questionnaire C30 (QLQ-C30) from the European Organization for Research and Treatment of Cancer before randomization and every 8 weeks thereafter. ${ }^{13}$ After progression during a chemotherapy sequence, the choice of second-line and additional treatment was at the discretion of the investigator.

\section{Outcomes}

The primary end point was TCD, defined as the time elapsed between randomization and tumor progression during a chemotherapy sequence. Patients who did not progress during the chemotherapy sequence were censored at their last follow-up within the protocol or censored at the initiation of second line of chemotherapy or other therapeutic strategy. Patients with R0 resection or R1 resection without chemotherapy were censored at the date of surgery, and patients lost to follow-up were censored at the date of last news.

Secondary end points were PFS, defined as the time between randomization and first progression or death by any cause, and time to treatment failure, defined as the time between randomization and definitive discontinuation of the protocol treatment, whatever the cause. Patients alive and still under treatment were censored at date of last news; OS, defined as the time between randomization and death by any cause; duration of the first CFI, defined as the time between the end of induction chemotherapy and the first reintroduction of chemotherapy or the date of the last injection in the maintenance arm; total duration of CFIs, defined as the sum of the duration of successive CFI; objective response rate, defined according to RECIST 1.1 criteria; toxicity; and QoL assessed by the QLQC30 form completed at each evaluation. ${ }^{11}$

Because the randomization was performed before the induction chemotherapy, a significant number of patients were not fit enough to be subject to the CFI and reintroduction strategy. Since our study was designed, two other trials have been published, which showed that only $56 \%$ and $65 \%$ of patients screened before induction were enrolled into a trial designed to include maintenance or CFI. ${ }^{7,14}$ Thus, we decided to perform an exploratory per-protocol analysis to describe our strategy in the subgroup of patients eligible for maintenance or CFI. The per-protocol population was defined as patients with at least one reintroduction of chemotherapy subsequent to a first disease progression during CFI.

\section{Statistical Analyses}

All efficacy analyses were based on a modified intent-to-treat analysis, defined as patients who had received at least one dose of the treatment, and a per-protocol analysis. TCD, OS, and PFS were analyzed using the unstratified Kaplan-Meier method. Treatment arms were compared using the conventional log-rank test. Sensitivity analyses with the stratified Cox model on the resected tumor and Köhne criteria (stratification factors at baseline) were also performed for TCD, OS, and PFS. The hypothesis was to extend from 10 to 14 months the median TCD with the maintenance of bevacizumab during CFI (hazard ratio [HR], 0.714). With a two-sided $\alpha$ risk of $5 \%$, and a power of $90 \%, 396$ events were required. Taking into account an interim analysis (IA) at 50\% of the events and an assumption of 10 inclusions per month, and with a percentage of patients lost to followup of $10 \%, 492$ patients had to be enrolled. ${ }^{11}$ The data collected were analyzed at the Centre de Randomization, Gestion Analyze of the Fédération Francophone de Cancérologie Digestive. The follow-up ended in December 2016. Final analyses were performed on a database frozen in January 2017. 


\section{RESULTS}

Between March 2010 and July 2013, 494 patients were randomly assigned to either FOLFIRI plus bevacizumab induction chemotherapy followed by bevacizumab maintenance during CFI ( $\mathrm{n}=247$ ) or to the same induction chemotherapy followed by observation during CFI ( $\mathrm{n}=247)$ (Fig 1). An IA was performed as planned in the protocol after 203 events had been observed in March 2014. The IA showed no significant improvement of TCD with maintenance therapy. At the time of this analysis, the recruitment was completed so the IA had no effect on it. Investigators continued the follow-up of their patients according to the protocol. Baseline patient characteristics were well balanced between the two treatment groups (Table 1). The median follow-up was 51.2 (interquartile range [IQR], 48.8 to 58.8 ) months in the maintenance arm and 54.9 (IQR, 50.1 to 60.6) months in the observation arm. Six patients still remain in treatment (three in the maintenance arm and three in the observation arm), and 94 patients $(19.3 \%)$ are alive, $46(18.8 \%)$ in the maintenance arm and 48 $(19.8 \%)$ in the observation arm.

Disease progression or death during induction chemotherapy occurred in 85 patients (17\%), 39 (15.9\%) in the maintenance arm and $46(18.9 \%)$ in the observation arm. Chemotherapy reinduction after first progression during CFI was performed in $64.7 \%$ of the patients, who had not experienced progression during induction chemotherapy (60.2\% in the maintenance arm and $69.5 \%$ in the observation arm). Eighty-two patients in the maintenance arm and 60 patients in the observation arm were alive or without progression after induction chemotherapy but did not undergo reinduction of FOLFIRI plus bevacizumab (Fig 1). The percentage of patients able to receive a second sequence of chemotherapy was not significantly different between the two arms, but more patients received a third sequence in the observation arm (Table 2). The median total number of chemotherapy cycles was 13 (IQR, 12 to 20 ) in the maintenance arm and 16 (IQR, 12 to 24) in the observation arm.

The median TCD was 15 months in both arms (HR, 1.07; 95\% CI, 0.85 to $1.34 ; P=.57$; Fig $2 \mathrm{~A}$ ). The median PFS was 9.2 months in the maintenance arm and 8.9 months in the observation arm (HR, 0.91 ; 95\% CI, 0.76 to $1.09 ; P=.316$; Fig 2B). The 12-month PFS was $30.2 \%( \pm 2.9)$ in the maintenance arm and $21.01( \pm 2.6)$ in the observation arm. The median time to treatment failure was 11.1 months in the maintenance arm and 12.1 months in the observation arm (HR, $1.17 ; 95 \% \mathrm{CI}, 0.97$ to $1.40 ; P=.092)$. The median duration of first CFI was 4.1 months in the maintenance

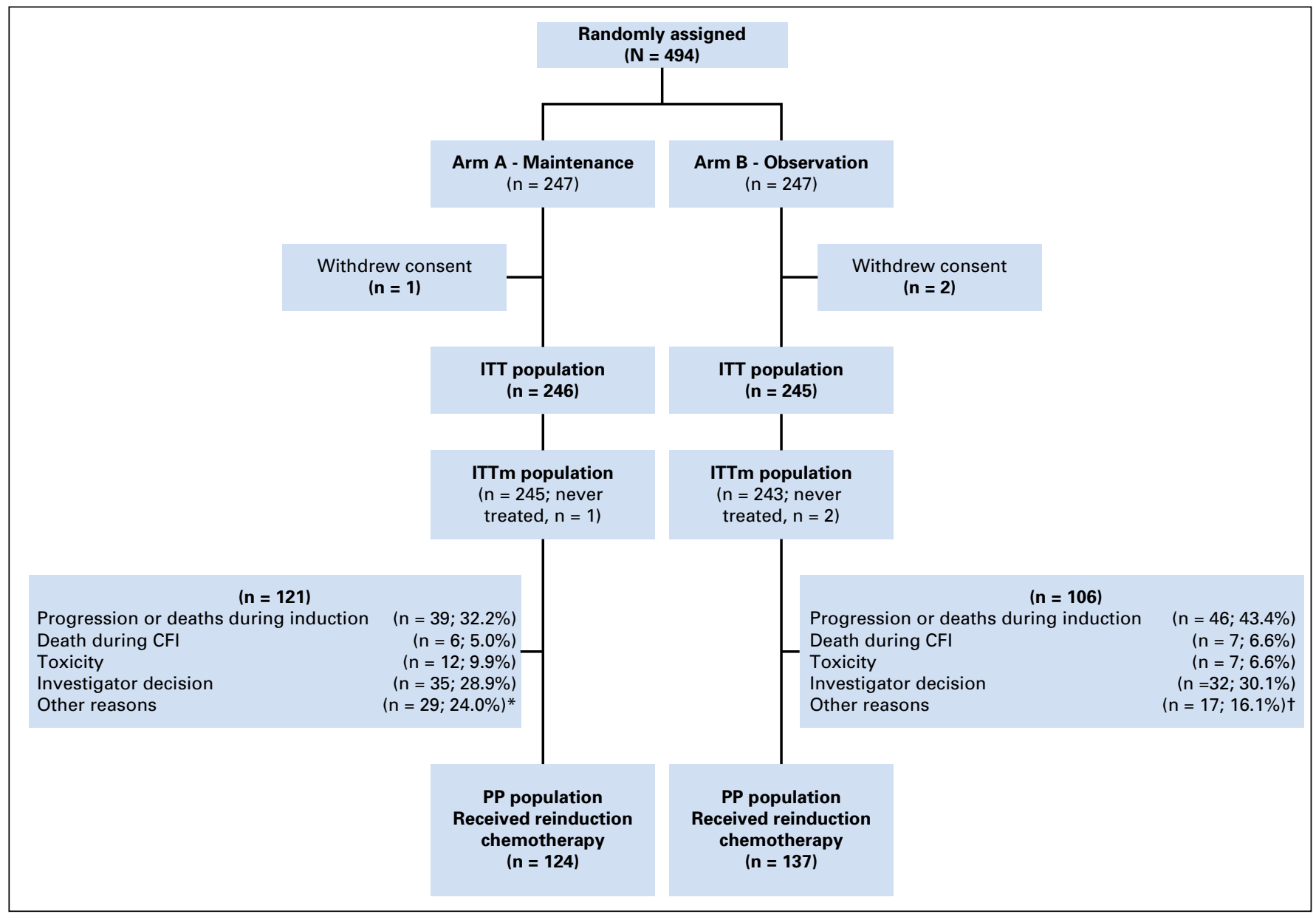

Fig 1. Flow chart. CFI, chemotherapy-free intervals; ITT, intent to treat; ITTm; modified intent to treat; PP, per protocol; PS, performance status. $\left({ }^{*}\right)$ Other reasons:

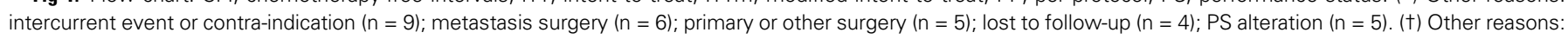
intercurrent event or contra-indication $(n=6)$; metastasis surgery $(n=2)$; primary or other surgery $(n=6)$; lost to follow-up $(n=2)$; PS alteration ( $n=1)$. 


\begin{tabular}{|c|c|c|}
\hline & Maintenance & Observation \\
\hline Characteristic & $(n=245)$ & $(n=243)$ \\
\hline Median age, years $(\mathrm{Q} 1, \mathrm{Q} 2)$ & $64.2(57.3,72.3)$ & $65.0(57.8,72.7)$ \\
\hline Male gender, No. (\%) & $152(62.0)$ & $164(67.5)$ \\
\hline Primary tumor resected, No. (\%) & $141(57.8)$ & $140(57.6)$ \\
\hline \multicolumn{3}{|l|}{ WHO performance status, No. (\%) } \\
\hline 0 & $118(48.2)$ & $115(47.3)$ \\
\hline 1 & $112(45.7)$ & $104(42.8)$ \\
\hline 2 & $15(6.1)$ & $24(9.9)$ \\
\hline \multicolumn{3}{|l|}{ Köhne criteria, No. (\%) } \\
\hline Low & 97 (39.6) & 84 (34.6) \\
\hline Intermediate & $104(42.4)$ & $112(46.1)$ \\
\hline High & $44(18.0)$ & 47 (19.3) \\
\hline \multicolumn{3}{|l|}{ Number of metastatic sites, No. (\%) } \\
\hline 1 & $103(42.0)$ & 89 (36.6) \\
\hline$>1$ & $142(58.0)$ & $154(63.4)$ \\
\hline \multicolumn{3}{|l|}{ Location ( $n=356$ ), No. (\%) } \\
\hline Right colon & $58(41.7)$ & $42(35.0)$ \\
\hline Left colon & $80(57.6)$ & $77(64.2)$ \\
\hline Rectum & $43(17.8)$ & $56(23.9)$ \\
\hline Median CEA $(n=473), \mu \mathrm{g} / \mathrm{L}(\mathrm{Q} 1, \mathrm{Q} 3)$ & $66.4(12.2,525.1)$ & $59.4(8.5,372.0)$ \\
\hline $\begin{array}{l}\text { Median CA } 19.9(n=468), \mathrm{U} / \mathrm{mL} \\
(\mathrm{Q} 1, \mathrm{Q} 2)\end{array}$ & $87.0(11.0,700.0)$ & $70.5(15.0,656.0)$ \\
\hline Mutated KRAS ( $\mathrm{n}=375)$ & $85(45.7)$ & $88(46.6)$ \\
\hline Mutated BRAF $(\mathrm{n}=245)$ & $12(9.6)$ & $9(7.5)$ \\
\hline
\end{tabular}

Abbreviations: CA, cancer antigen; CEA, carcinoembryonic antigen; $\mathrm{Q}$, quartile.

arm and 4.2 in the observation arm. The median total duration of cumulative CFI was 4.9 months in the maintenance arm and 5.5 months in the observation arm, but in the case of patients with at least two successive CFIs, these values were 8.6 months and 10.7 months, respectively. The median OS was 21.7 months in maintenance arm and 22 months in the observation arm (HR, $1.07 ; 95 \%$ CI, 0.88 to $1.29 ; P=.500$; Fig 2 C). The best response was a partial or complete tumor response in $53.0 \%$ of patients in the maintenance arm and $56.5 \%$ of patients in the observation arm. Second-line treatment and the $\mathrm{R} 0$ resection rate were similar in both arms (Table 2).

The per-protocol analysis was performed on 261 patients (53\% of all enrolled patients). In this population, the median TCD was 17.8 months in the maintenance arm and 23.3 months in the observation arm (HR, 1.16; 95\% CI, 0.86 to $1.57 ; P=.339)$. The PFS was 9.9 months in the maintenance arm and 9.5 months in the observation arm (HR, $0.89 ; 95 \% \mathrm{CI}, 0.70$ to $1.13 ; P=.339)$. The median duration of first CFI was 4.3 months in both arms. The median OS was 27.6 months in the maintenance arm and 28.5 months in the observation arm (HR, 1.11; 95\% CI, 0.86 to $1.45 ; P=.424)$.

Univariable analyses for TCD, PFS, and OS are presented in the Appendix (Tables A1, A2, and A3, online only). Multivariable analysis revealed that female gender, WHO performance status $\geq 2$, and unresected primary tumor were associated with a shorter TCD. WHO performance status $\geq 2$, unresected primary tumor, and age older than 65 years were associated with a shorter OS (Table 3).

Results of the subgroup of patients with tumor BRAF status available are listed in Appendix Table A4. Subgroup analysis revealed no condition favoring bevacizumab maintenance for

\begin{tabular}{|c|c|c|c|}
\hline Treatment & $\begin{array}{c}\text { Maintenance } \\
(n=245) \\
\text { No. }(\%)\end{array}$ & $\begin{array}{c}\text { Observation } \\
\text { (n=243) } \\
\text { No. }(\%)\end{array}$ & $P$ \\
\hline No reintroduction & $121(49.4)$ & $106(43.6)$ & .034 \\
\hline One sequence postinduction & 78 (31.8) & 76 (31.3) & \\
\hline Two sequences postinduction & $28(11.4)$ & $23(9.5)$ & \\
\hline $\begin{array}{l}\text { Three sequences or more } \\
\text { postinduction }\end{array}$ & $18(7.3)$ & 38 (15.6) & \\
\hline Second-line & $159(65)$ & $158(65)$ & \\
\hline Chemotherapy alone & $53(22)$ & $58(24)$ & \\
\hline Chemotherapy plus anti-VEGF & $77(31)$ & 77 (32) & \\
\hline Chemotherapy plus anti-EGFR & $29(12)$ & $23(9)$ & \\
\hline Ro surgery & $15(6)$ & $10(4.1)$ & .700 \\
\hline
\end{tabular}

either TCD (Appendix Fig A1, online only), PFS (Appendix Fig A2, online only), or OS (Appendix Fig A3, online only).

Treatment-related adverse events of grades 3, 4, and 5 were observed in $80 \%$ of patients in the maintenance arm and $79 \%$ of patients in the observation arm (Table 4). More toxicities, and especially cardiovascular toxicities, were observed in the maintenance arm during the CFI. QoL was assessed only in 162 patients in the maintenance arm and 173 patients in the observation arm. No significant difference was observed between arms. The median time to QoL global degradation was 14.7 (95\% CI, 11.9 to 18.1 ) months in the maintenance arm and 14.4 (95\% CI, 12.7 to 19.4 ) months in the observation arm. It was 3.7 (95\% CI, 2.3 to 5.2 ) months and 5.1 (95\% CI, 3.6 to 8.0 ) months for the physical functional subscale and 19.8 (95\% CI, 17.8 to 24.1$)$ months and 21.5 (95\% CI, 15.4 to 21.1 ) months for the asthenia subscale in the maintenance and observation arms, respectively.

\section{DISCUSSION}

The PRODIGE 9 trial investigated bevacizumab alone as maintenance treatment after induction chemotherapy and after each reinduction sequence. This is the first trial to have addressed this strategy in a large number of patients treated with irinotecan-based induction chemotherapy. This strategy resulted in a unexpected prolonged TCD in both arms compared with our initial hypotheses. ${ }^{11}$ Our trial failed to demonstrate a difference in TCD between the two treatment strategies of administering and not administering bevacizumab monotherapy during the maintenance period. In the AIO 0207 trial, the time to failure of the strategy after induction chemotherapy did not differ significantly between the bevacizumab monotherapy arm (6.1 months) and the observation arm (6.4 months). ${ }^{7}$ Thus, the results of both trials are concordant. In the AIO 0207 trial, PFS after induction chemotherapy was slightly but significantly improved by bevacizumab compared with observation $(4.6 v 3.5$ months; $P=.0018) .{ }^{7}$ In the SAKK $41 / 06$ trial, the time to progression was 4.1 months in the bevacizumab arm and 2.9 in the observation arm, but the difference was not significant. ${ }^{8}$ The computed tomography or magnetic resonance imaging scans were performed every 6 weeks in the AIO 0207 and SAKK 41/06 trials, but every 8 weeks in the PRODIGE 9 trial. Thus, 


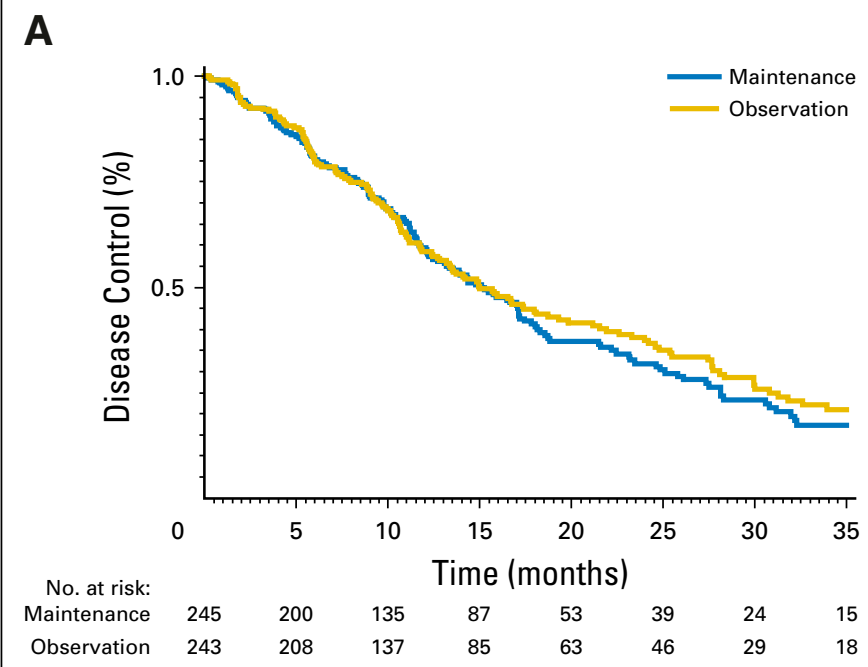

B

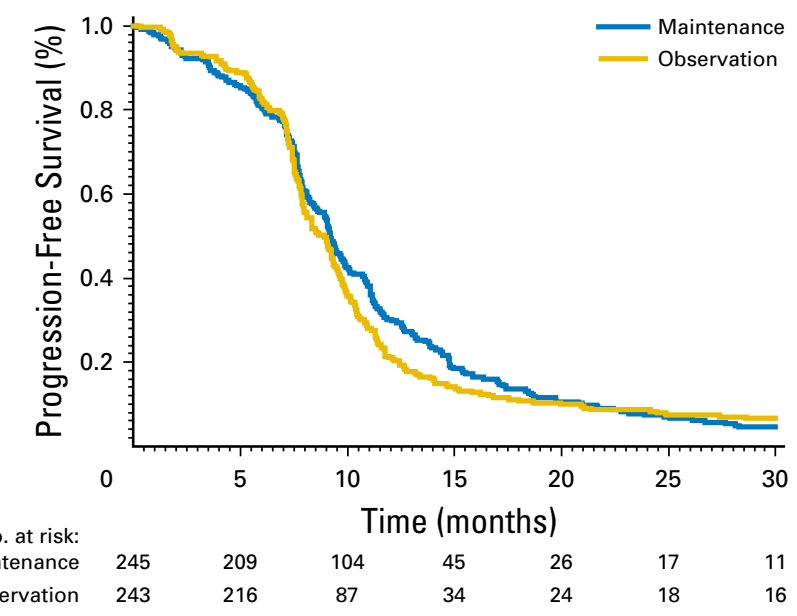

C

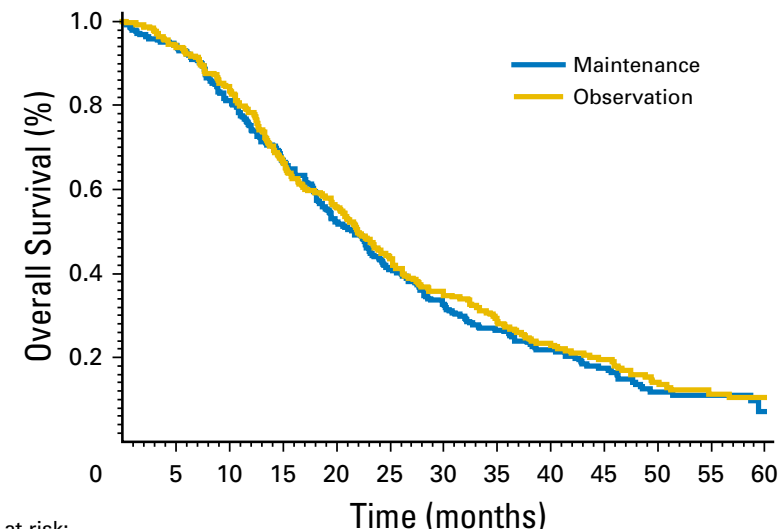

No. at risk:

$\begin{array}{lllllllllllllll}\text { Maintenance } & 245 & 231 & 199 & 163 & 127 & 99 & 79 & 64 & 48 & 34 & 19 & 9 & 5\end{array}$

$\begin{array}{llllllllllllll}\text { Observation } & 243 & 228 & 204 & 162 & 135 & 105 & 84 & 67 & 51 & 38 & 23 & 13 & 9\end{array}$

Fig 2. Kaplan-Meier curves according to study treatment. (A) Tumor control duration. (B) Progression-free survival. (C) Overall survival.

it cannot be ruled out that the PRODIGE 9 trial design was unable to detect a small difference in the time to first progression between patients treated with bevacizumab and those in the observation group.

The OS was not modified by bevacizumab monotherapy compared with observation after induction chemotherapy in the PRODIGE 9 trial as in the two previous randomized trials that made this comparison. ${ }^{7,8}$ Furthermore, in our trial, subgroup exploratory analyses did not find any subgroup benefitting from bevacizumab maintenance treatment. Altogether, it could be concluded that bevacizumab monotherapy after induction chemotherapy in mCRC has little or no beneficial effect.

It is suspected that discontinuation of bevacizumab promotes a rebound in cancer growth. Our results show that bevacizumab

\begin{tabular}{|c|c|c|c|}
\hline Prognostic Factors & $\begin{array}{l}\operatorname{TCD}(n=487) \\
\operatorname{HR}(95 \% \mathrm{Cl})\end{array}$ & $\begin{array}{l}\text { PFS }(n=487) \\
\text { HR }(95 \% \text { CI) }\end{array}$ & $\begin{array}{l}\text { OS }(n=487) \\
\text { HR }(95 \% \mathrm{Cl})\end{array}$ \\
\hline Sex (women $v$ men) & $1.29(1.01$ to 1.63$) ; P=.038$ & - & - \\
\hline WHO performance status $(2 \vee 0)$ & $\begin{array}{l}2.84(1.59 \text { to } 5.06) ; \\
\quad P<.001 \text { (overall } P=.002 \text { ) }\end{array}$ & $\begin{array}{l}2.02(1.23 \text { to } 3.31) \\
\quad P=.005 \text { (overall } P=.02)\end{array}$ & $\begin{array}{l}2.53(1.53 \text { to } 4.18) ; \\
\quad P<.001 \text { (overall } P=.0015)\end{array}$ \\
\hline Primary tumor resected (no $v$ yes) & $1.34(1.06$ to 1.70$) ; P=.014$ & $1.15(0.95$ to 1.39$) ; P=.140$ & $1.26(1.03$ to 1.54$) ; P=.027$ \\
\hline Age $(>65 v \leq 65$ years $)$ & - & - & $1.34(1.10$ to 1.64$) ; P=.004$ \\
\hline Number of metastatic sites $(>1 \vee 1)$ & - & - & $2.18(1.05$ to 4.56$) ; P=.037$ \\
\hline
\end{tabular}

Abbreviations: HR, hazard ratio; OS, overall survival; PFS, progression-free survival; TCD, tumor control duration. 


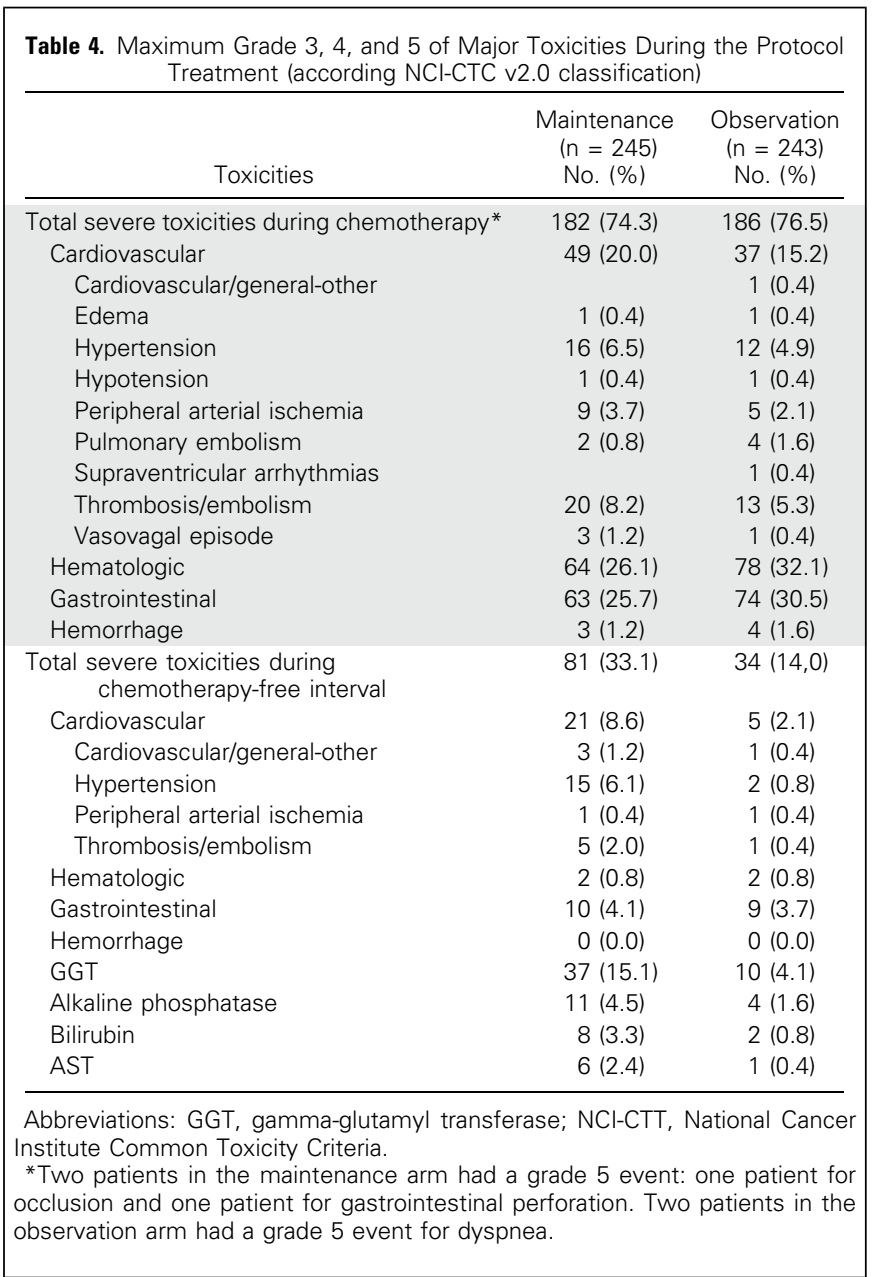

monotherapy maintenance did not delay reprogression. From a health care economic point of view, avoiding unnecessary bevacizumab treatment can result in substantial cost savings.

Several large phase III trials addressed the question of maintenance therapy previously. ${ }^{3,7,9}$ Comparison of OS across trials is difficult because of different trial schedules and times of randomization, which occurred before or after induction chemotherapy; in addition, we enrolled only patients with definitively nonresectable metastases and patients were not selected according to RAS mutation, contrary to other recent phase III trials. ${ }^{15,16}$ The question of a detrimental effect of CFI could be raised but was not assessed by the PRODIGE 9 trial. The patient selection rules to give a maintenance treatment or a CFI without any treatment deserve additional evaluation. Moreover, patients with BRAF mutations should probably not be enrolled in a de-escalation strategy because of their distinct biology and a poor prognosis compared with the rest of the population.

Chemotherapy reinduction after first progression during CFI was performed in $53 \%$ of the modified intent-to-treat population, but this proportion reached $69.5 \%$ in the subgroup of patients with no disease progression during induction chemotherapy in the observation arm. This is a higher proportion than in the observation arm of the AIO 0207 trial (36\%) but comparable to that in the CAIRO 3 trial (58\%). In these previous trials, as in PRODIGE 9, investigator decision is frequently observed and suggests that some investigators are reluctant to use the reinduction strategy. The planned duration of induction chemotherapy was 24 weeks in the AIO 0207 trial, 18 weeks in the CAIRO 3 trial, and 24 weeks in the PRODIGE 9 trial. Given the lack of cumulative toxicity, especially neurotoxicity, reinduction using irinotecan-based chemotherapy may be more feasible than using oxaliplatin-based chemotherapy. It was possible to perform several reinductions of chemotherapy in some patients, resulting in a prolonged TCD. It is noteworthy that fewer successive reinductions of chemotherapy took place in the maintenance arm. Although there is not a clear explanation for this finding, it may have affected the primary end point. We observed more toxicities and especially cardiovascular toxicities in the maintenance arm during the CFI. This is in line with the result observed with the bevacizumab monotherapy arm in the study by Giantonio et al, ${ }^{10}$ with more than $30 \%$ of patients with any grade 3 to 4 toxicity and more than $8 \%$ with cardiovascular grade 3 to 4 toxicity. This increased toxicity in the maintenance arm might partly explain the lack of reinduction of chemotherapy in this arm. Nevertheless, we could not rule out that part of this increasing toxicity could be a result of more intensive monitoring of adverse events in the maintenance arm.

In the PRODIGE 9 trial, unresected primary tumor, WHO performance status $>2, B R A F$ mutation, and more than one metastatic site were associated with a shorter OS. Tumor BRAF mutation was also reported as a poor prognostic factor in previous trials. ${ }^{7,17}$ This finding encourages the conduct of a specific trial in this subgroup of patients. An unresected primary tumor was associated with poor OS in several mCRC first-line chemotherapy trials. ${ }^{18}$ Moreover, in the CAIRO 3 trial, a better OS was observed in patients with metachronous metastases and in patients with synchronous metastases but with a resected primary tumor than in those without a resected primary tumor. Interestingly, in this trial, the OS benefit of maintenance chemotherapy with bevacizumab compared with observation was mainly obtained in patients with synchronous metastases but with a resected primary tumor. ${ }^{9}$

A poorer prognosis was reported for patients with a rightsided tumor compared with those with a left-sided tumor. ${ }^{19}$ In the PRODIGE 9 trial, univariable analysis showed a right-sided tumor to be significantly associated with a poor OS compared with a rectal tumor, but this was not demonstrated in multivariable analysis. However, age older than 65 years was associated with a poor prognosis in multivariable analysis. Thus, age seemed to be more related to prognosis than primary location. However, the prognostic value of primary location seemed more important when therapy targeting anti-epidermal growth factor receptor rather than bevacizumab was used as front-line treatment. ${ }^{20}$ Nevertheless, all the results derived from primary location should be viewed with caution because the analysis was a subgroup post hoc analysis.

In previous trials that have compared maintenance chemotherapy with observation after induction chemotherapy combined with bevacizumab, maintenance treatment had no significant effect on OS. $^{7-9}$ Thus, observation or maintenance chemotherapy with fluoropyrimidine combined with bevacizumab are valuable options. Moreover, in the PRODIGE 9 trial, we observed that the strategy of reinduction with irinotecan-based chemotherapy combined with bevacizumab resulted in a prolonged TCD and OS. 
In conclusion, the PRODIGE 9 trial demonstrated that bevacizumab monotherapy has no effect when used as maintenance treatment after induction chemotherapy. Additional research is needed to better define subgroups of patients who should receive maintenance chemotherapy after induction treatment or could undergo a true chemotherapy-free interval.

\section{AUTHORS' DISCLOSURES OF POTENTIAL CONFLICTS OF INTEREST}

Disclosures provided by the authors are available with this article at jco.org.

\section{AUTHOR CONTRIBUTIONS}

Conception and design: Thomas Aparicio, Karine Le Malicot, Jaafar Bennouna

Provision of study materials or patients: Thomas Aparicio, François Ghiringhelli, Valérie Boige, Julien Taieb, Olivier Bouché, Jean-Marc Phelip, Eric François, Christian Borel, Roger Faroux, Laetitia Dahan, Stéphane Jacquot, Dominique Genet, Faiza Khemissa, Etienne Suc, Françoise Desseigne, Patrick Texereau, Come Lepage, Jaafar Bennouna

Collection and assembly of data: Karine Le Malicot

Data analysis and interpretation: All authors

Manuscript writing: All authors

Final approval of manuscript: All authors

Accountable for all aspects of the work: All authors

\section{REFERENCES}

1. Grothey A, Sargent D: Overall survival of patients with advanced colorectal cancer correlates with availability of fluorouracil, irinotecan, and oxaliplatin regardless of whether doublet or single-agent therapy is used first line. J Clin Oncol 23:9441-9442, 2005

2. Hurwitz $H$, Fehrenbacher L, Novotny $W$, et al: Bevacizumab plus irinotecan, fluorouracil, and leucovorin For metastatic colorectal cancer. N Engl J Med 350:2335-2342, 2004

3. Chibaudel B, Maindrault-Goebel F, Lledo G, et al: Can chemotherapy be discontinued in unresectable metastatic colorectal cancer? The GERCOR OPTIMOX2 Study. J Clin Oncol 27:5727-5733, 2009

4. Labianca R, Sobrero A, Isa L, et al: Intermittent versus continuous chemotherapy in advanced colorectal cancer: A randomised 'GISCAD' trial. Ann Oncol 22:1236-1242, 2011

5. Tournigand $C$, Cervantes $A$, Figer $A$, et al: OPTIMOX1: A randomized study of FOLFOX4 or FOLFOX7 with oxaliplatin in a stop-and-go fashion in advanced colorectal cancer-A GERCOR study. J Clin Oncol 24:394-400, 2006

6. Díaz-Rubio E, Gómez-España A, Massutí B, et al: First-line XELOX plus bevacizumab followed by XELOX plus bevacizumab or single-agent bevacizumab as maintenance therapy in patients with metastatic colorectal cancer: The phase III MACRO TTD study. Oncologist 17:15-25, 2012

7. Hegewisch-Becker S, Graeven U, Lerchenmüller $\mathrm{CA}$, et al: Maintenance strategies after first-line oxaliplatin plus fluoropyrimidine plus bevacizumab for patients with metastatic colorectal cancer (AIO 0207): A randomised, non-inferiority, open-label, phase 3 trial. Lancet Oncol 16: 1355-1369, 2015

8. Koeberle D, Betticher DC, von Moos R, et al: Bevacizumab continuation versus no continuation after first-line chemotherapy plus bevacizumab in patients with metastatic colorectal cancer: A randomized phase III non-inferiority trial (SAKK 41/06). Ann Oncol 26:709-714, 2015

9. Simkens $L H$, van Tinteren $H$, May $A$, et al: Maintenance treatment with capecitabine and bevacizumab in metastatic colorectal cancer (CAIRO3): A phase 3 randomised controlled trial of the Dutch Colorectal Cancer Group. Lancet 385:1843-1852, 2015

10. Giantonio BJ, Catalano PJ, Meropol NJ, et al: Bevacizumab in combination with oxaliplatin, fluorouracil, and leucovorin (FOLFOX4) for previously treated metastatic colorectal cancer: Results from the Eastern Cooperative Oncology Group Study E3200. J Clin Oncol 25:1539-1544, 2007

11. Aparicio $T$, Linot $B$, Le Malicot $K$, et al: FOLFIRI +bevacizumab induction chemotherapy followed by bevacizumab or observation in metastatic colorectal cancer, a phase III trial (PRODIGE 9-FFCD 0802). Dig Liver Dis 47:271-272, 2015

12. Köhne $\mathrm{CH}$, Cunningham $\mathrm{D}$, Di Costanzo $\mathrm{F}$ et al: Clinical determinants of survival in patients with 5-fluorouracil-based treatment for metastatic colorectal cancer: Results of a multivariate analysis of 3825 patients. Ann Oncol 13:308-317, 2002

13. Aaronson NK, Ahmedzai S, Bergman B, et al: The European Organization for Research and Treatment of Cancer QLQ-C30: A quality-of-life instrument for use in international clinical trials in oncology. J Nat Cancer Inst 85:365-376, 1993

14. Tournigand $C$, Chibaudel $B$, Samson $B$, et al: Bevacizumab with or without erlotinib as maintenance therapy in patients with metastatic colorectal cancer (GERCOR DREAM; OPTIMOX3): A randomised, open-label, phase 3 trial. Lancet Oncol 16: 1493-1505, 2015

15. Heinemann V, von Weikersthal LF, Decker T, et al: FOLFIRI plus cetuximab versus FOLFIRI plus bevacizumab as first-line treatment for patients with metastatic colorectal cancer (FIRE-3): A randomised, open-label, phase 3 trial. Lancet Oncol 15:1065-1075, 2014

16. Venook AP, Niedzwiecki D, Lenz HJ, et al: Effect of first-line chemotherapy combined with cetuximab or bevacizumab on overall survival in patients with KRAS wild-type advanced or metastatic colorectal cancer: A randomized clinical trial. JAMA 317:2392-2401, 2017

17. Cremolini C, Loupakis F, Antoniotti $C$, et al: FOLFOXIRI plus bevacizumab versus FOLFIRI plus bevacizumab as first-line treatment of patients with metastatic colorectal cancer: Updated overall survival and molecular subgroup analyses of the open-label, phase 3 TRIBE study. Lancet Oncol 16:1306-1315, 2015

18. Faron M, Pignon JP, Malka D, et al: Is primary tumour resection associated with survival improvement in patients with colorectal cancer and unresectable synchronous metastases? A pooled analysis of individual data from four randomised trials. Eur J Cancer 51:166-176, 2015

19. Missiaglia $E$, Jacobs $B$, D'Ario $G$, et al: Distal and proximal colon cancers differ in terms of molecular, pathological, and clinical features. Ann Oncol 25:1995-2001, 2014

20. Venook A, Niedzwiecki D, Innocenti F. Impact of primary tumor location on overall survival and progression-free survival in patients with metastatic colorectal cancer: Analysis of CALGB/ SWOG 80405 (Alliance). J Clin Oncol 34, 2016 (suppl; abstr 3504)

\section{Affiliations}

Thomas Aparicio, Centre Hospitalier Universitaire (CHU) Saint Louis, Assistance Public Hôpitaux de Paris (APHP), and Université Paris 7, Sorbonne Paris Cité; Julien Taieb, Hôpital Européen Georges Pompidou, Paris; Francois Ghiringhelli, Centre Georges-François Leclerc; Karine Le Malicot, Fédération Francophone de Cancérologie Digestive; Come Lepage, CHU Le Bocage, Institut National de la Santé et de la Recherche Médicale Unité Mixte de Recherche (UMR) 1231, Dijon; Valérie Boige, Institut Gustave Roussy, Villejuif; Olivier Bouché, CHU Robert Debré, Reims; Jean-Marc Phelip, CHU Saint Etienne-Hôpital Nord, Saint Priest en Jarez; Eric François, Centre Antoine Lacassagne, Nice; Christian Borel, Paul Strauss Center, Strasbourg; Roger Faroux, Centre Hospitalier (CH) La Roche sur Yon, La Roche sur Yon; Laetitia Dahan, CHU La Timone, Aix-Marseille-University, Marseille; Stéphane Jacquot, Centre de Cancérologie du Grand Montpellier, Montpellier; Dominique Genet, Accompagnement de la Recherche Clinique Hospitalière (ARCH)/Polyclinique, Limoges; Faiza Khemissa, CH Saint Jean, Perpignan; Etienne Suc, Clinique Saint Jean du Languedoc, Toulouse; Françoise Desseigne, Centre Léon Bérard, Lyon; Patrick Texereau, CH Layne, Mont-De-Marsan; and Jaafar Bennouna, Institut de Cancérologie de l'Ouest, Saint Herblay, France. 


\section{Support}

Supported by Fédération Francophone de Cancérologie Digestive (FFCD), which was responsible for the study management; for the design and conduct of the study; for collection, management, analysis, and interpretation of the data; for preparation, review, or approval of the manuscript; and for the decision to submit the manuscript for publication. The FFCD thanks the Ligue Nationale Contre le Cancer, who provided financial support to FFCD. Hoffmann-La Roche provided financial support for study management (Grant No. ML28232).

\section{Prior Presentation}

Presented at the American Society of Clinical Oncology 2016 Annual Meeting, Chicago, IL, June 3-7, 2016.

\section{JCO-Exceptionally Quick Review Process}

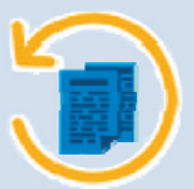

JCO offers a Rapid Review and Rapid Communications program that publishes novel, timely research online within 4-6 weeks of the original submission date. This is made possible by a dynamic panel of editors with far-ranging specialties, supported by an immense database of respected scientific reviewers and biostatisticians.

Submit to JCO: please visit http://submit.jco.org 
Bevacizumab Maintenance Versus No Maintenance During Chemotherapy-Free Intervals in Metastatic Colorectal Cancer: A Randomized Phase III Trial (PRODIGE 9)

The following represents disclosure information provided by authors of this manuscript. All relationships are considered compensated. Relationships are self-held unless noted. I = Immediate Family Member, Inst = My Institution. Relationships may not relate to the subject matter of this manuscript. For more information about ASCO's conflict of interest policy, please refer to www.asco.org/rwc or ascopubs.org/jco/site/ifc.

Thomas Aparicio

Consulting or Advisory Role: Ipsen, Pierre Fabre, HalioDx, Bristol-Myers Squibb

Speakers' Bureau: Roche, Pfizer, LEO Pharma, Amgen, MSD Oncology

Research Funding: Roche (Inst), Bayer (Inst), Novartis (Inst)

Travel, Accommodations, Expenses: Ipsen Pharma, Novartis Oncology, Roche, Hospira

\section{François Ghiringhelli}

No relationship to disclose

Valérie Boige

Consulting or Advisory Role: Merck Serono, Bayer, Amgen, Sanofi,

Daiichi Sankyo, Novartis, Roche, Prestizia

\section{Karine Le Malicot}

No relationship to disclose

Julien Taieb

Consulting or Advisory Role: Roche, Merck KGaA, Amgen, Celgene, Eli Lilly, Shire, Servier, Sirtex

Speakers' Bureau: Servier, Amgen, Shire, Roche/Genentech, Sanofi, Merck, Eli Lilly

Olivier Bouché

Honoraria: Novartis

Consulting or Advisory Role: Roche, Merck, Amgen, Bayer, Eli Lilly

Jean-Marc Phelip

Honoraria: Roche, Sanofi, Eli Lilly, Merck, Amgen

Consulting or Advisory Role: Eli Lilly, Roche, Roche (Inst), Merck (Inst)

Eric François

Honoraria: Eli Lilly, Novartis, Amgen

Consulting or Advisory Role: Roche, Merck, Sanofi

Travel, Accommodations, Expenses: Roche, Servier, Merck

\section{Christian Borel}

Honoraria: Merck

Consulting or Advisory Role: AstraZeneca, Bristol-Myers Squibb

Travel, Accommodations, Expenses: Merck

\section{Roger Faroux}

Consulting or Advisory Role: Amgen, Merck, Roche

Research Funding: Merck

Travel, Accommodations, Expenses: Amgen, Celgene, Merck, Roche

\section{Laetitia Dahan}

Consulting or Advisory Role: Amgen, Baxalta, Celgene, Eli Lilly, Merck, Sanofi, Roche

Research Funding: Ipsen, Eli Lilly, MSD, Sanofi

Travel, Accommodations, Expenses: Celgene, Ipsen, Sanofi

Stéphane Jacquot

No relationship to disclose

Dominique Genet

Research Funding: Novartis Oncology, Pfizer, Roche

Travel, Accommodations, Expenses: Amgen, Pfizer, Roche

Faiza Khemissa

Speakers' Bureau: Sanofi Pasteur, Roche

Travel, Accommodations, Expenses: Roche, Ipsen, Sanofi, Fresinus

\section{Etienne Suc}

Consulting or Advisory Role: Pfizer

Travel, Accommodations, Expenses: Celgene, Novartis Oncology, Pfizer, Roche

\section{Françoise Desseigne}

No relationship to disclose

Patrick Texereau

No relationship to disclose

\section{Come Lepage}

Honoraria: Novartis, Eli Lilly, Bayer

Consulting or Advisory Role: Advanced accelerator applications, HalioDx

Travel, Accommodations, Expenses: Amgen, Novartis, Ipsen, Bayer

\section{Jaafar Bennouna}

Honoraria: Roche, Boehringer Ingelheim, MSD Oncology, AstraZeneca, Bristol-Myers Squibb

Consulting or Advisory Role: Roche, Boehringer Ingelheim, AstraZeneca, MSD Oncology, BMS

Travel, Accommodations, Expenses: Roche, BMS 


\section{Acknowledgment}

We thank all participating patients and their families, and the study groups and investigators from the participating countries (Appendix). We also thank the team from the Fédération Francophone de Cancérologie Digestive data center.

\section{Appendix}

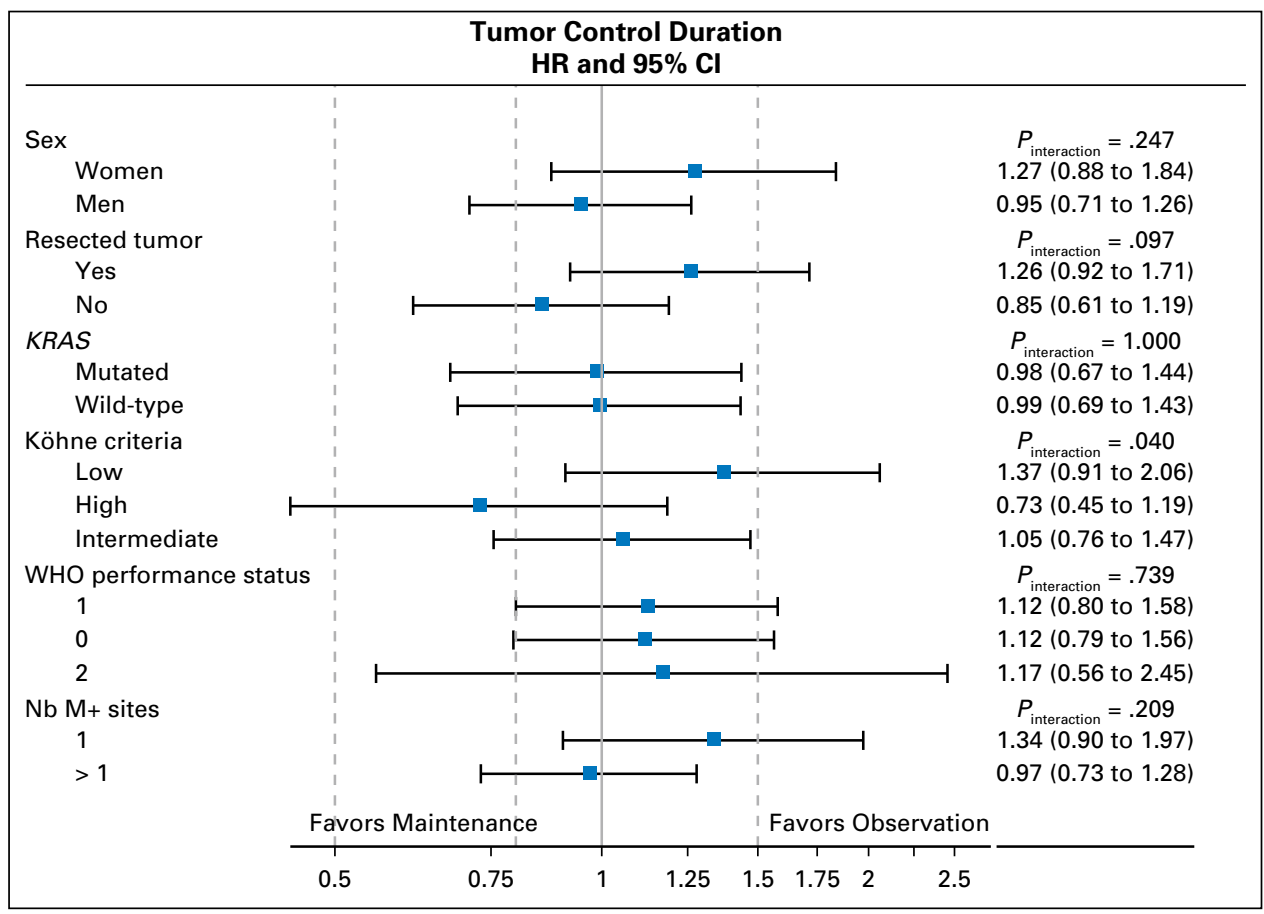

Fig A1. Subgroup analysis for tumor control duration. 


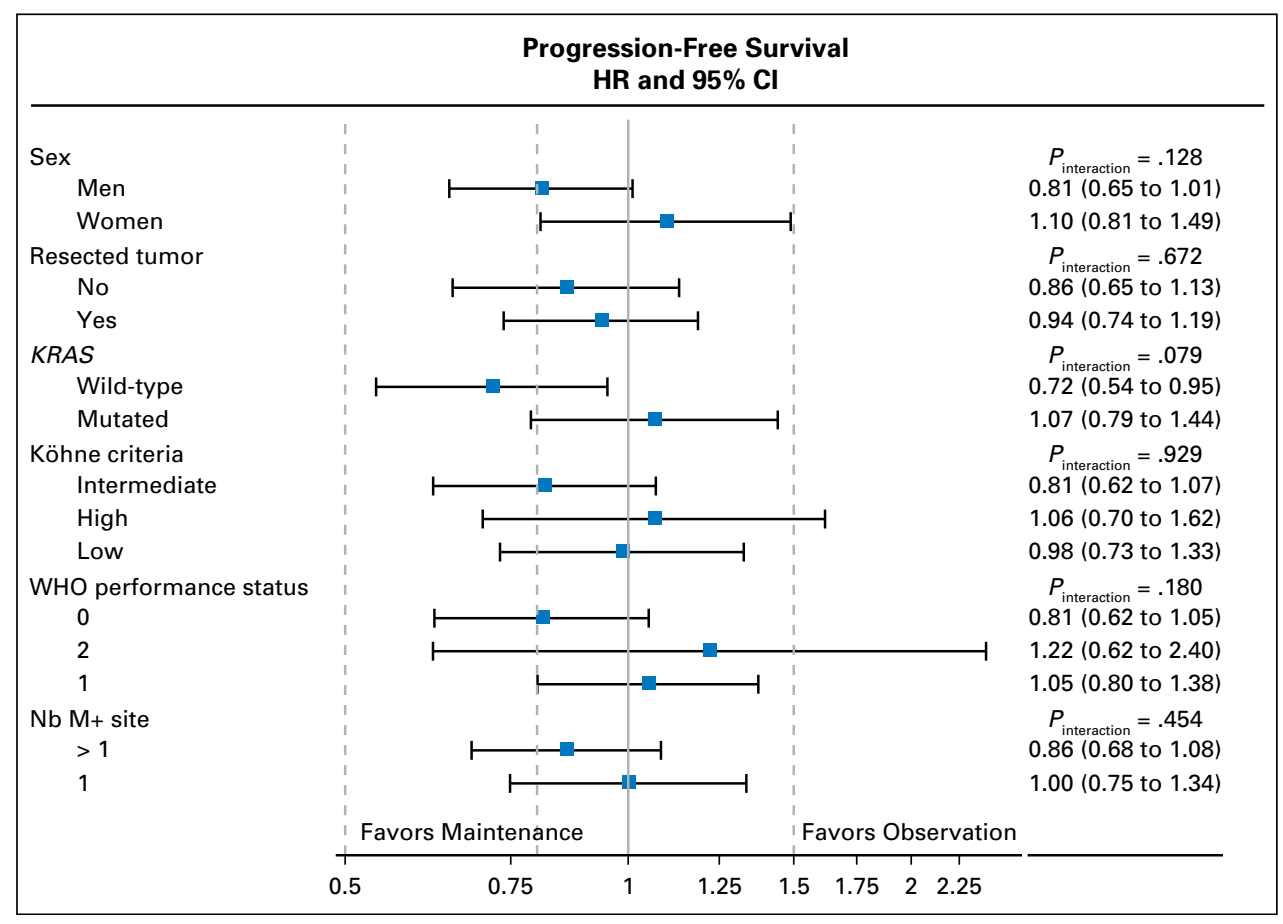

Fig A2. Subgroup analysis for progression-free survival.

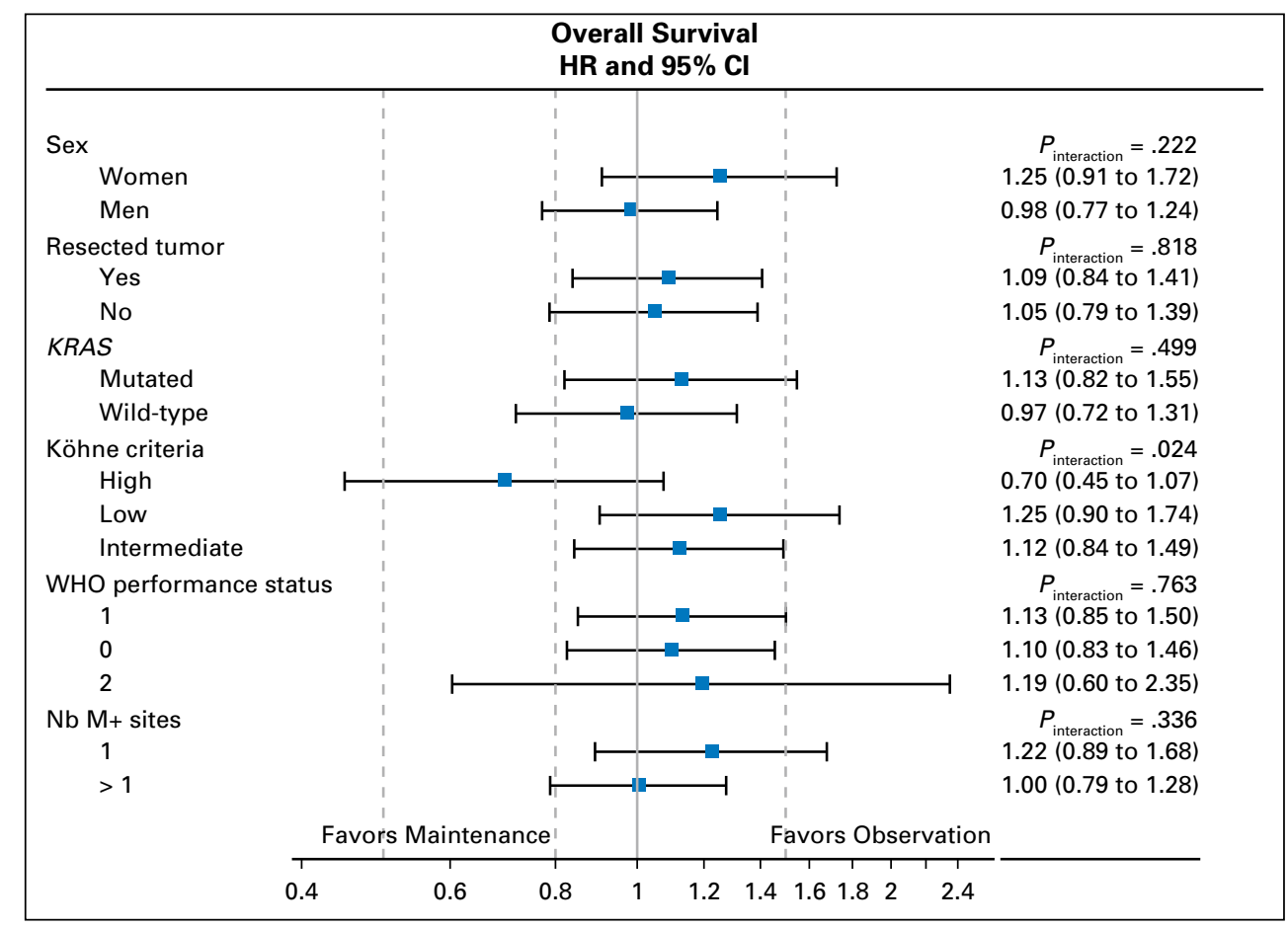

Fig A3. Subgroup analysis for overall survival. 
Table A1. Univariable Analysis of Prognostic Factors Associated With Tumor Control Duration

\begin{tabular}{|c|c|c|c|c|}
\hline \multicolumn{2}{|c|}{ Prognostic Factor } & $\frac{\mathrm{n} / \mathrm{N} \text { Event }}{151 / 245}$ & $\frac{\text { \% Event }}{61.63}$ & \multirow{2}{*}{$\frac{\mathrm{HR}(95 \% \mathrm{Cl}) ; P}{1.07\left(\begin{array}{c}(0.85 \text { to } 1.34) ; .573 \\
\text { Reference }\end{array}\right.}$} \\
\hline & B (observation) & $151 / 243$ & 62.14 & \\
\hline Sex & Women & $115 / 172$ & 66.86 & 1.30 (1.03 to 1.64$) ; .026$ \\
\hline \multirow[t]{2}{*}{ Age } & $\leq 65$ years & $150 / 249$ & 60.24 & Reference \\
\hline & $>65$ years & $152 / 239$ & 63.60 & 1.11 (0.88 to 1.39$) ; .3724$ \\
\hline Köhne criteria & Low & 96/181 & 53.04 & Reference \\
\hline \multirow{2}{*}{ WHO performance status } & 1 & $134 / 216$ & 62.04 & 1.33 (1.04 to 1.69 ); .020 \\
\hline & 2 & $33 / 39$ & 84.62 & $5.12(3.43 \text { to } 7.65)_{i}<.001$ \\
\hline \multirow[t]{2}{*}{ Primary tumor resected } & No & $141 / 206$ & 68.45 & 1.55 (1.24 to 1.95$) ;<.001$ \\
\hline & Yes & $160 / 281$ & 56.94 & Reference \\
\hline \multirow[t]{2}{*}{ No. of metastatic sites } & 1 & 105/192 & 54.69 & Reference \\
\hline & $>1$ & $197 / 296$ & 66.55 & 1.28 (1.01 to 1.62$) ; .043$ \\
\hline \multirow{2}{*}{ Localization } & Left colon & $94 / 157$ & 59.87 & 1.11 (0.80 to 1.52$) ; .537$ \\
\hline & Rectum & $63 / 99$ & 63.64 & Reference \\
\hline
\end{tabular}

Abbreviation: HR, hazard ratio.

Table A2. Univariable Analysis of Prognostic Factors Associated With Progression-Free Survival

\begin{tabular}{|c|c|c|c|c|}
\hline \multicolumn{2}{|c|}{ Prognostic Factor } & \multirow{2}{*}{$\frac{n / N \text { Event }}{240 / 245}$} & \multirow{2}{*}{$\frac{\% \text { Event }}{97.96}$} & \multirow{3}{*}{ 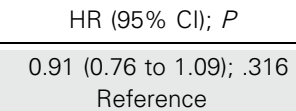 } \\
\hline Treatment arm & A (maintenance) & & & \\
\hline & B (observation) & $237 / 243$ & 97.53 & \\
\hline \multirow[t]{2}{*}{ Sex } & Women & $167 / 172$ & 97.09 & $0.96(0.80$ to 1.16$) ; .686$ \\
\hline & Men & $310 / 316$ & 98.10 & Reference \\
\hline \multirow[t]{2}{*}{ Age } & $\leq 65$ years & $244 / 249$ & 97.99 & Reference \\
\hline & $>65$ years & $233 / 239$ & 97.49 & $0.90(0.75$ to 1.08$) ; .257$ \\
\hline \multirow[t]{3}{*}{ Köhne criteria } & Low & $175 / 181$ & 96.69 & Reference \\
\hline & High & $91 / 91$ & 100.00 & 2.12 (1.64 to 2.75$) ;<.001$ \\
\hline & Intermediate & $211 / 216$ & 97.69 & 1.16 (0.95 to 1.42$) ; .148$ \\
\hline \multirow[t]{3}{*}{ WHO performance status } & 0 & $226 / 233$ & 97.00 & Reference \\
\hline & 1 & $212 / 216$ & 98.15 & $1.14(0.95$ to 1.38$) ; .169$ \\
\hline & 2 & $39 / 39$ & 100.00 & $3.05(2.16$ to 4.31$) ;<.001$ \\
\hline \multirow[t]{2}{*}{ Primary tumor resected } & No & $203 / 206$ & 98.54 & 1.25 (1.04 to 1.51$) ; .015$ \\
\hline & Yes & $273 / 281$ & 97.15 & Reference \\
\hline \multirow[t]{2}{*}{ No. of metastatic sites } & 1 & $186 / 192$ & 96.88 & Reference \\
\hline & $>1$ & $291 / 296$ & 98.31 & $1.26(1.05$ to 1.52$) ; .014$ \\
\hline \multirow[t]{2}{*}{ KRAS status } & Mutant & $172 / 173$ & 99.42 & $1.07(0.87$ to 1.32$) ; .509$ \\
\hline & Wild-type & $195 / 202$ & 96.53 & Reference \\
\hline \multirow[t]{2}{*}{$B R A F$ status } & Mutant & $20 / 21$ & 95.24 & 1.60 (1.01 to 2.54$) ; .046$ \\
\hline & Wild-type & $218 / 224$ & 97.32 & Reference \\
\hline \multirow[t]{3}{*}{ Localization } & Right colon & $99 / 100$ & 99.00 & $1.17(0.89$ to 1.55$) ; .266$ \\
\hline & Left colon & $151 / 157$ & 96.18 & $1.02(0.79$ to 1.31$) ; .887$ \\
\hline & Rectum & $97 / 99$ & 97.98 & Reference \\
\hline
\end{tabular}

Abbreviation: HR, hazard ratio. 
Aparicio et al

\begin{tabular}{|c|c|c|c|c|}
\hline \multicolumn{2}{|c|}{ Prognostic Factor } & \multirow{2}{*}{$\frac{n / N \text { Event }}{214 / 245}$} & \multirow{2}{*}{$\frac{\% \text { Event }}{87.35}$} & \multirow{3}{*}{$\frac{\mathrm{HR}(95 \% \mathrm{Cl}) ; P}{1.07 \begin{array}{c}(0.88 \text { to } 1.29) ; .500 \\
\text { Reference }\end{array}}$} \\
\hline Treatment arm & A (maintenance) & & & \\
\hline & B (observation) & $209 / 243$ & 86.01 & \\
\hline \multirow[t]{2}{*}{ Sex } & Women & $153 / 172$ & 88.95 & 1.09 (0.89 to 1.32$) ; .415$ \\
\hline & Men & $270 / 316$ & 85.44 & Reference \\
\hline \multirow[t]{2}{*}{ Age } & $\leq 65$ years & $213 / 249$ & 85.54 & Reference \\
\hline & $>65$ years & $210 / 239$ & 87.87 & 1.28 (1.05 to 1.54$) ; .013$ \\
\hline \multirow[t]{3}{*}{ Köhne criteria } & Low & 146/181 & 80.66 & Reference \\
\hline & High & $88 / 91$ & 96.70 & 2.94 (2.24 to 3.87$) ;<.001$ \\
\hline & Intermediate & $189 / 216$ & 87.50 & $1.22(0.98$ to 1.52$) ; .073$ \\
\hline \multirow[t]{3}{*}{ WHO performance status } & 0 & $193 / 233$ & 82.83 & Reference \\
\hline & 1 & $192 / 216$ & 88.89 & 1.30 (1.06 to 1.58$) ; .011$ \\
\hline & 2 & $38 / 39$ & 97.44 & 4.26 (2.98 to 6.09$)_{i}<.001$ \\
\hline \multirow[t]{2}{*}{ Primary tumor resected } & No & $191 / 206$ & 92.72 & 1.40 (1.15 to 1.69 ); .001 \\
\hline & Yes & $231 / 281$ & 82.21 & Reference \\
\hline \multirow[t]{2}{*}{ No. of metastatic sites } & 1 & 156/192 & 81.25 & Reference \\
\hline & $>1$ & $267 / 296$ & 90.20 & 1.39 (1.14 to 1.70$) ; .001$ \\
\hline \multirow[t]{2}{*}{ KRAS status } & Mutant & $153 / 173$ & 88.44 & 1.16 (0.93 to 1.45$) ; .183$ \\
\hline & Wild-type & $169 / 202$ & 83.66 & Reference \\
\hline \multirow[t]{2}{*}{$B R A F$ status } & Mutant & $19 / 21$ & 90.48 & 1.73 (1.07 to 2.77$) ; .024$ \\
\hline & Wild-type & $187 / 224$ & 83.48 & Reference \\
\hline \multirow[t]{3}{*}{ Localization } & Right colon & $87 / 100$ & 87.00 & 1.39 (1.03 to 1.89$) ; .031$ \\
\hline & Left colon & $133 / 157$ & 84.71 & 1.11 (0.85 to 1.47$) ; .440$ \\
\hline & Rectum & $83 / 99$ & 83.84 & Reference \\
\hline
\end{tabular}

\begin{tabular}{|c|c|c|c|}
\hline Prognostic Factor & $\operatorname{TCD}(\mathrm{n}=245), \mathrm{HR}(95 \% \mathrm{Cl}) ; P$ & PFS $(n=245), H R(95 \% \mathrm{Cl}) ; P$ & OS $(n=245), \operatorname{HR}(95 \% \mathrm{Cl}) ; P$ \\
\hline BRAF status (mutated $v$ wild-type) & 2.29 (1.27 to 4.14$): .006$ & 1.77 (1.07 to 2.92$) ; .03$ & 2.08 (1.26 to 3.44$) ; .004$ \\
\hline WHO performance status $(2 \vee 0)$ & $\begin{array}{l}2.48(1.14 \text { to } 5.41) ; .02 \\
\text { (overall } P=.06)\end{array}$ & $\begin{array}{l}2.69(1.32 \text { to } 5.51) ; .007 \\
\text { (overall } P=.01)\end{array}$ & $\begin{array}{l}2.34(1.11 \text { to } 4.91) ; .03 \\
\text { (overall } P=.05)\end{array}$ \\
\hline Primary tumor resected (no $v$ yes) & $1.44(1.02$ to 2.04$) ; .038$ & - & 1.53 (1.14 to 2.06$) ; .005$ \\
\hline No. of metastatic sites $(>1 \vee 1)$ & - & - & 4.24 (1.16 to 15.49$) ; .03$ \\
\hline
\end{tabular}




\section{List of Investigators/Collaborators}

Véronique Guérin-Meyer, MD (Centre Paul Papin, Angers); Benjamin Linot, MD (Centre Paul Papin, Angers); François-Xavier Caroli-Bosc, MD (Centre Hospitalier Universitaire Hôtel Dieu, Angers), Caroline Couffon, MD (Centre Hospitalier Universitaire Hôtel Dieu, Angers); Dominique Luet, MD (Centre Hospitalier Universitaire Hôtel Dieu, Angers); Guillaume Roquin, MD (Centre Hospitalier Universitaire Hôtel Dieu, Angers); Anne-Laure Villing, MD (Centre Hospitalier, Auxerre); Dominique Sevin-Robiche, MD (Clinique Sainte Marguerite, Auxerre); Franck Audemar, MD (Centre Hospitalier de la Côte Basque, Bayonne); Anne CadierLagnes, MD (Centre Hospitalier de la Côte Basque, Bayonne); Anne-Claire Dupont-Gossart, MD (Centre Hospitalier Universitaire Jean Minjoz, Besançon); Mohamed Ramdani, MD (Centre Hospitalier, Béziers); Yann Le Bricquir, MD (Centre Hospitalier, Béziers); Dany Gargot, MD (Centre Hospitalier, Blois); Thomas Aparicio, MD (Centre Hospitalier Universitaire Avicenne, Bobigny); Yves Becouarn, MD (Institut Bergonié, Bordeaux); Dominique Bechade, MD (Institut Bergonié, Bordeaux); Vincent Bourgeois, MD (Centre Hospitalier Duchenne, Boulogne sur Mer); Corinne Sarda, MD (Centre Hospitalier, Castres); Nathalie Hess-Laurens, MD (Centre Hospitalier, Castres); Jean-Marie Vantelon, MD (Centre Leonard de Vinci, Dechy); Claire Giraud, MD (Centre Leonard de Vinci, Dechy); François Ghiringhelli, MD (Centre Georges-François Leclerc, Dijon); Véronique Lorgis, MD (Centre Georges-François Leclerc, Dijon); Julie Vincent, MD (Centre Georges-François Leclerc, Dijon); Jean-Louis Jouve, MD (Centre Hospitalier le Bocage, Dijon); Laurent Bedenne, MD (Centre Hospitalier le Bocage, Dijon); Paul-Arthur Haineaux, MD (Centre Hospitalier le Bocage, Dijon); Côme Lepage, MD (Centre Hospitalier le Bocage, Dijon); Geneviève Boilleau-Jolimoy, MD (Centre de Radiothérapie du Parc, Dijon); Anne-Marie Queuniet, MD (Centre Hospitalier, Elbeuf); Stéfanie Oddou-Lagraniere, MD (Centre Hospitalier Chicas, Gap); François Dewaele, MD (Centre Hospitalier les Oudairies, La Roche-sur-Yon); Thierry Chatellier, MD (Centre Hospitalier les Oudairies, La Roche-sur-Yon); Roger Faroux, MD (Centre Hospitalier les Oudairies, La Roche-sur-Yon); Guillaume Medinger, MD (Centre Hospitalier les Oudairies, La Roche-sur-Yon); Anne Thirot-Bidault, MD (Centre Hospitalier Universitaire Bicêtre, Le Kremlin Bicêtre); Oana Cojocarasu, MD (Centre Hospitalier, Le Mans); Florence Kikolski, MD (Centre Hospitalier Robert Boulin, Libourne); Philippe Martin, MD (Centre Bourgogne, Lille); Dominique Genet, MD (Clinique François Chénieux, Limoges); Joël Ezenfis, MD (Centre Hospitalier, Longjumeau); Joëlle Egreteau, MD (Centre Hospitalier Bretagne Sud, Lorient); Isabelle Cumin, MD (Centre Hospitalier Bretagne Sud, Lorient); Régine Lamy, MD (Centre Hospitalier Bretagne Sud, Lorient); Julien Forestier, MD (Hôpital Edouard Herriot, Lyon); Catherine Lombard-Bohas, MD (Hôpital Edouard Herriot, Lyon); Yann Molin, MD (Hôpital Edouard Herriot, Lyon); Thomas Walter, MD (Hôpital Edouard Herriot, Lyon); Christelle De La Fouchardière, MD (Centre Léon Bérard, Lyon); Françoise Desseigne, MD (Centre Léon Bérard, Lyon); Denis Peré-Vergé, MD (Centre Hospitalier Saint Joseph, Lyon); Jean-Christophe Souquet, MD (Centre Hospitalier Universitaire de La Croix Rousse, Lyon); Lionel Wander, MD (Centre Hospitalier Universitaire de La Croix Rousse, Lyon); Yves Rinaldi, MD (Hôpital Européen, Marseille); Nicolas Barrière, MD (Hôpital Européen, Marseille); Mohamed Gasmi, MD (Hôpital Nord, Marseille); Muriel Duluc, MD (Centre Hospitalier Universitaire La Timone, Marseille); Jean-François Seitz, MD (Centre Hospitalier Universitaire La Timone, Marseille); Emmanuelle Norguet-Monnereau, MD (Centre Hospitalier Universitaire La Timone, Marseille); Laétitia Dahan, MD (Centre Hospitalier Universitaire La Timone, Marseille); Christophe Locher, MD (Centre Hospitalier, Meaux); Hatem Salloum, MD (Centre Hospitalier, Meaux); Farah Zerouala-Boussaha, MD (Centre Hospitalier, Meaux); Patrick Texereau, MD (Centre Hospitalier Layné, Mont de Marsan); Jérôme Dauba, MD (Hôpital Layné, Mont de Marsan); Miguel Carreiro, MD (Centre Hospitalier, Montauban); Nathalie Gérardin, MD (Centre Hospitalier, Montauban); Ahmed Azzedine, MD (Centre Hospitalier, Montélimar); Stéphane Jacquot, MD (Institut du cancer du Val d'Aurelle, Montpellier); Catherine Becht, MD (Institut du cancer du Val d'Aurelle, Montpellier); Eric Janssen, MD (Institut du cancer du Val d'Aurelle, Montpellier); Lafforgue Béatrice, MD (Institut du cancer du Val d'Aurelle, Montpellier); Eric François, MD (Centre Antoine Lacassagne, Nice); Philippe Follana, MD (Centre Antoine Lacassagne, Nice); Abakar Mahamat, MD (Centre Hospitalier Universitaire l'Archet, Nice); Suzanne Nguyen, MD (Centre Hospitalier, Beauvais); Jacques Cretin, MD (Clinique Valdegour, Nîmes); Jérôme Meunier, MD (Centre Hospitalier de la Source, Orléans); Corina Cornila, MD (Centre Hospitalier de la Source, Orléans); Gaël Goujon, MD (Centre Hospitalier Universitaire Bichat, Paris); Bruno Landi, MD (Hôpital Européen Georges Pompidou, Paris); Julien Taieb, MD (Hôpital Européen Georges Pompidou, Paris); Philippe Rougier, MD (Hôpital Européen Georges Pompidou, Paris); Isabelle Trouilloud, MD (Hôpital Européen Georges Pompidou, Paris); Aziz Zaanan, MD (Hôpital Européen Georges Pompidou, Paris); Céline Lepère, MD (Hôpital Européen Georges Pompidou, Paris); Jean-Baptiste Bachet, MD (Centre Hospitalier Pitié Salpêtrière, Paris); Touraj Mansourbakht, MD (Centre Hospitalier Pitié Salpêtrière, Paris); Jean-Marc Gornet, MD (Centre Hospitalier Universitaire Saint-Louis, Paris); Laurent Cany, MD (Polyclinique Francheville, Périgueux); Charles-Briac Levaché, MD (Polyclinique Francheville, Périgueux); Faiza Khemissa, MD (Centre Hospitalier Saint Jean, Perpignan); Sophie Pesque-Penaud, MD (Centre Hospitalier Universitaire Haut-Lévêque, Pessac); Eric Terrebonne, MD (Centre Hospitalier Universitaire Haut-Lévêque, 
Pessac); Julien Vergniol, MD (Centre Hospitalier Universitaire Haut-Lévêque, Pessac); Pierre-Luc Etienne, MD (Centre Cario, PlérinSur-Mer); Mathieu Baconnier, MD (Centre Hospitalier Annecy Genevois, Pringy); Emmanuel Maillard, MD (Centre Hospitalier Annecy Genevois, Pringy); Laetitia Stefani, MD (Centre Hospitalier Annecy Genevois, Pringy); Olivier Bouché, MD (Centre Hospitalier Universitaire Robert Debré); Julien Volet, MD (Centre Hospitalier Universitaire Robert Debré); Marie-Claude Gouttebel, MD (Centre Hospitalier Drôme Nord, Romans-sur-Isère); Marc Porneuf, MD (Centre Hospitalier Yves Le Foll, Saint-Brieuc); Vincent Quentin, MD (Centre Hospitalier Yves Le Foll, Saint-Brieuc); Jean-Marc Phelip, MD (Hôpital Bellevue, Saint-Etienne); Xavier Coulaud, MD (Hôpital Bellevue, Saint-Etienne); Jaafar Bennouna, MD (Institut de Cancérologie de l'Ouest, Saint-Herblain); Hélène Senellart, MD (Institut de Cancérologie de l'Ouest, Saint-Herblain); Sandrine Hiret, MD (Institut de Cancérologie de l'Ouest, SaintHerblain); Jean-Yves Douillard, MD (Institut de Cancérologie de l'Ouest, Saint-Herblain); Hélène Castanie, MD (Institut de Cancérologie de l'Ouest, Saint-Herblain); Laurent Gasnault, MD (Centre Joliot Curie, Saint Martin Boulogne); Thierry Chatellier, MD (Clinique Mutualiste de l'Estuaire, Saint Nazaire); Ivan Graber, MD (Clinique Trenel, Saint Colombe les Vienne); Annunziato Alessio, MD (Clinique Trenel, Saint Colombe les Vienne); Meher Ben Abdelghani, MD (Centre Paul Strauss, Strasbourg); Christian Borel, MD (Centre Paul Strauss, Strasbourg); Christine Belletier, MD (Centre Paul Strauss, Strasbourg); Etienne Suc, MD (Clinique Saint Jean du Languedoc, Toulouse); Thierry Lecomte, MD (Centre Hospitalier Trousseau, Tours); Etienne Dorval-Danquechin, MD (Centre Hospitalier Trousseau, Tours); Zied Ladhib, MD (Centre Hospitalier, Valence); Marie-Christine Kaminsky, MD (Institut de Cancérologie de Lorraine, Vandoeuvre Les Nancy); Thierry Conroy, MD (Institut de Cancérologie de Lorraine, Vandoeuvre Les Nancy); Lynn Rob, MD (Institut de Cancérologie de Lorraine, Vandoeuvre Les Nancy); Valérie Boige, MD (Institut Gustave Roussy, Villejuif); David Malka, MD (Institut Gustave Roussy, Villejuif); Pascal Burtin, MD (Institut Gustave Roussy, Villejuif); Elie Zrihen, MD (Institut Gustave Roussy, Villejuif); Michel Ducreux, MD (Institut Gustave Roussy, Villejuif); Antoine Hollebecque, MD (Institut Gustave Roussy, Villejuif). 